\title{
Moderate therapeutic efficacy of positron emission tomography-navigated repetitive transcranial magnetic stimulation for chronic tinnitus: a randomised, controlled pilot study
}

\author{
C Plewnia, M Reimold, A Najib, G Reischl, S K Plontke, C Gerloff
}

See Editorial Commentary, p 113

J Neurol Neurosurg Psychiatry 2007;78:152-156. doi: 10.1136/jnnp.2006.095612

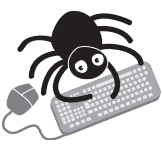

Additional figure is available at http://innp.bmijournals. com/supplemental

See end of article for authors' affiliations

Correspondence to:

C Plewnia, Department of Psychiatry, Brain-Stimulation Laboratory, University of Tuebingen, Osianderstrasse 24, D-72076 Tuebingen, Germany; christian. plewnia@uni-tuebingen.de

Received 13 April 2006 Revised 3 July 2006

Accepted 19 July 2006

Published Online First

4 August 2006
Background: Tinnitus has been shown to respond to modulations of cortical activity by high-frequency and low-frequency repetitive transcranial magnetic stimulation (rTMS).

Objective: To determine the tinnitus-attenuating effects of a 2-week daily regimen of rTMS, navigated to the maximum of tinnitus-related increase in regional cerebral blood flow.

Methods: Six patients with chronic tinnitus were enrolled in this sham-controlled crossover study and treated with $2 \times 2$ weeks of suprathreshold $1 \mathrm{~Hz}$ rTMS $(30 \mathrm{~min}$ ) applied to the region with maximal tinnitus-related increase in regional cerebral blood flow delineated by functional imaging with [ $\left.{ }^{15} \mathrm{O}\right] \mathrm{H}_{2} \mathrm{O}$ positron emission tomography and a control area. Tinnitus-related distress was assessed before and after each treatment and 2 weeks after the end of the 4 -week course of stimulation using a validated tinnitus questionnaire. Additional self-assessment scores of tinnitus change, loudness and annoyance were obtained.

Results: In five of six patients, rTMS induced greater reduction of the tinnitus questionnaire score than sham stimulation. In two patients, all parameters measured (tinnitus change score, tinnitus loudness, tinnitus annoyance) showed unequivocal improvement. At the group level, the degree of response in the tinnitus questionnaire score was correlated with tinnitus-associated activation of the anterior cingulate cortex. Two weeks after the final stimulation, tinnitus had returned to baseline in all patients but one.

Conclusion: Tinnitus can be attenuated by low-frequency rTMS navigated to each person's maximum tinnitusrelated cortical hyperactivity. The effects are only moderate; interindividual responsiveness varies and the attenuation seems to wear off within 2 weeks after the last stimulation session. Notably, tinnitus-related anterior cingulate cortex activation seems to predict the response to rTMS treatment.
$\mathrm{T}$ innitus is the phantom perception of sound or noise in the absence of an auditory stimulus and is a common symptom of disorders of the auditory system. ${ }^{1}$ Its chronic form affects between $5 \%$ and $15 \%$ of the general population. ${ }^{2}$ In $1-3 \%$ of the population, it causes severe impairment of the quality of life. ${ }^{3}$ In most cases, tinnitus is associated with hearing loss that is often induced by noise exposure or is age related.' Nevertheless, currently no specific pharmacological treatments are available that provide a replicable, long-term effect on tinnitus superior to placebo. The use of antidepressants, anticonvulsants and benzodiazepines may offer relief to some patients, but these treatments are largely considered palliative rather than curative. Hearing aids or electronic devices, producing a white noise that covers up the annoying perception, can be of help. ${ }^{5}{ }^{6}$ The combination of noise generators and counselling is called "tinnitus retraining therapy" and is often used in the management of chronic tinnitus. ${ }^{7}$ Although psychology-based strategies effectively support the habituation and adaptation to tinnitus, ${ }^{8}$ the development of treatments is constrained by the limited pathophysiological knowledge.

In recent years, it has become widely accepted that maladaptive changes of central information processing are critically involved in tinnitus perception and generation. Particularly, studies on positron emission tomography (PET) have provided evidence for an association between tinnitus and activation of areas involved in the perception and processing of sounds and speech. ${ }^{910}$ In these studies, regional cerebral blood flow ( $\mathrm{rCBF}$ ) during tinnitus perception was contrasted with rCBF when tinnitus was transiently reduced by lidocaine injection, ${ }^{911}{ }^{12}$ oral facial movements ${ }^{13}$ or gaze. ${ }^{10}{ }^{14}$ These data indicate that tinnitus corresponds to abnormally high levels of regional cortical activity, which would increase and decrease with tinnitus loudness. This is in line with animal studies indicating a reduction of intracortical inhibition due to deafferentation. ${ }^{15}{ }^{16}$ Nevertheless, imaging studies alone cannot warrant the behavioural relevance of the associated activation.

In the initial studies on transcranial magnetic stimulation (TMS), these areas were subjected to short trains of repetitive transcranial magnetic stimulation (rTMS), interfering with the neuronal activity in underlying areas. ${ }^{17}{ }^{18}$ Indeed, a shortlasting decrease in tinnitus was observed, providing evidence for the critical role of cortical auditory and association areas in tinnitus perception. In contrast with short trains of highfrequency rTMS, low-frequency rTMS is suited to induce a longer-lasting decrease in cortical activity in the stimulated area, as shown in the motor cortex. ${ }^{19}$ We have previously shown that this kind of stimulation can reduce tinnitus in a

Abbreviations: $A C C$, anterior cingulate cortex; $\mathrm{PET}$, positron emission tomography; rCBF, regional cerebral blood flow; rTMS, repetitive transcranial magnetic stimulation; SPM, statistical parametric mapping; TCS, tinnitus-change score; TMS, transcranial magnetic stimulation; $T Q$, tinnitus questionnaire 
dose-dependent manner for up to $30 \mathrm{~min}^{12}$ However, the clinical use of rTMS in tinnitus would require a persistent reduction in tinnitus loudness and its associated distress. A prior series of experiments has provided initial evidence for the efficacy and practicability of this treatment strategy. Repeated sessions of rTMS directed towards the auditory cortex were applied over 1 week in a placebo-controlled, crossover design. $^{2021}$ After real rTMS, Kleinjung et al ${ }^{21}$ reported a reduction of the mean tinnitus score by $7.5 \%$ (compared with baseline). Interestingly, after 6 months the reduction was even more pronounced $(12 \%)$.

The aim of this study was to test whether a 2-week series of low-frequency rTMS, guided to each patient's maximum of tinnitus-related cortical activity as assessed by $\left[{ }^{15} \mathrm{O}^{1} \mathrm{H}_{2} \mathrm{O}\right.$ PET, can induce a lasting suppression of tinnitus compared with the control stimulation of a non-cortical site eliciting equivalent noise and sensations.

\section{METHODS}

Six patients (one woman and five men, 49-68 years old) with chronic ( $>1$ year) bilateral tinnitus gave written, informed consent and were included in this controlled, crossover study that was conducted in accordance with the Declaration of Helsinki and approved by the local ethics committee. All subjects participated in a prior PET/rTMS study. ${ }^{12}$ Patients with heart disease, history of seizures or brain lesions, metal implants, cardiac pacemaker and current use of psychotherapeutic drugs were excluded from the study. Before experimental treatment with rTMS, all subjects underwent a standard otolaryngological physical examination and audiological testing, including a pure-tone audiogram and subjective tinnitus matching of loudness and frequency. ${ }^{22}$ The hearing level was characterised by the standard "four tone average" in pure tone audiometry and by audiogram shape. Audiogram shape was defined as being predominantly high-frequency hearing loss if the average (arithmetic mean) of hearing thresholds at 4, 6 and $8 \mathrm{kHz}$ was twice as high or more than the average of 0.5, 1 and $2 \mathrm{kHz}$. In pancochlear hearing loss, the audiometric threshold was increased throughout the entire frequency range (table 1).

To individually adjust the selection of the target area for rTMS, the pattern of tinnitus-related rCBF was obtained by $\left[{ }^{15} \mathrm{O}\right] \mathrm{H}_{2} \mathrm{O}$ PET in each subject. ${ }^{12}$ The scanning was carried out on 2 days, and each day before and after a lidocaine-induced reduction of tinnitus loudness. Lidocaine was injected over l min under electrocardiogram monitoring by a cardiologist, because of the risk of lidocaine-induced cardiac arrhythmia. Dynamic data were acquired after bolus injection of $1.8 \mathrm{~GB}$ $\left[{ }^{15} \mathrm{O}\right] \mathrm{H}_{2} \mathrm{O}$, with a GE Advance PET scanner (General Electrics Medical System, Milwaukee, USA; three-dimensional mode). As an index for tissue perfusion, the sum of counts (no decay correction) was computed from injection to $100 \mathrm{~s}$ after the turning point of the whole-brain time-activity curve.
The standard software of the scanner (FORE rebinning, two-dimensional filtered backprojection) was used to calculate the attenuation-corrected images. All images were realigned with statistical parametric mapping (SPM)2 (Wellcome Department of Cognitive Neurology, London, UK), and intensity normalised. Mean images (four scans) were calculated for anatomical orientation; difference images were computed after smoothing ( $10 \mathrm{~mm} \mathrm{G}$ ) and thresholded at $5 \%$ of mean cortical perfusion. The results of the group analysis $(n=9)$, including the present patients, were published previously. ${ }^{12}$

Mean and difference PET images were coregistered with structural magnetic resonance imaging and fed into the neuronavigation device (Brainsight; Magstim, Whitland, UK). This approach allowed guiding of the rTMS to the individual maximum of tinnitus-associated $\mathrm{rCBF}$ in the temporoparietal cortex (see supplemental figure at http://jnnp.bmjjournals.com/ supplemental). Two subjects ( 1 and 6 ) showed a symmetrical activation pattern in the posterior temporoparietal cortex. In both cases, we selected the more rostral area as the stimulation target. Brodmann areas were determined after spatial normalisation with SPM2. Stimulation was performed in a randomised, balanced and sham-controlled, crossover design with a TMS device for repetitive stimulation with biphasic pulses (Magstim Rapid; Magstim), with $1 \mathrm{~Hz}$ at $120 \%$ of the individual motor threshold for $30 \mathrm{~min}$ (1800 stimuli) on 20 consecutive working days (4 weeks). Motor threshold was defined as the minimal intensity necessary to evoke motor evoked potentials of $>50 \mu \mathrm{V}$ in a small hand muscle (musculus abductor poilicis brevis) in at least 5 of 10 stimuli. For sham stimulation, the coil was placed at the lower occiput at the same distance to the ear. In this way, the control stimulation was accompanied by a similar noise (between 60 and $75 \mathrm{~dB}$ ) and comparable aversive sensation (pricking, muscle twitches).

Neither loudness nor other psychoacoustic measures of tinnitus show a consistent relationship to perceived loudness of tinnitus, disability or annoyance. Therefore, we decided to not use psychoacoustic measures as the primary outcome criteria and no post-intervention scores were obtained. ${ }^{23}{ }^{24}$

As the primary outcome measure, the effect of rTMS on tinnitus was estimated by the validated German version of the tinnitus questionnaire (TQ ${ }^{25}$ originally developed by Hallam et al. ${ }^{26}$ This questionnaire is suitable for repeated measurement of tinnitus-related distress and was completed before $\left(\mathrm{TQ}_{0}\right)$ and then after every 2 weeks of either the verum or sham stimulation $\left(\mathrm{TQ}_{1}\right.$ and $\mathrm{TQ}_{2}$, respectively), as well as 2 weeks after the end of the 4-week course of treatment $\left(\mathrm{TQ}_{3}\right)$. Depending on the order of stimulation (verum, sham), $\mathrm{TQ}_{1}$ and $\mathrm{TQ}_{2}$ will be referred to as $\mathrm{TQ}_{\mathrm{VER}}$ and $\mathrm{TQ}_{\mathrm{SHA}}$. Response to rTMS was expressed as the percentage difference in tinnitus questionnaire: $\Delta \mathrm{TQ}=100 \% \times\left(\mathrm{TQ}_{\mathrm{VER}}-\mathrm{TQ}_{\mathrm{SHA}}\right) /\left(\left(\mathrm{TQ}_{\mathrm{VER}}+\mathrm{TQ}_{\mathrm{SHA}}\right) /\right.$ $2)$. The values of the tinnitus questionnaire (CONDITION, four levels: baseline, verum, control, 2 weeks after treatment) were

Table 1 Demographic data, audiometric measures and treatment conditions

\begin{tabular}{|c|c|c|c|c|c|c|c|c|}
\hline Subj & Age/sex & $\begin{array}{l}\text { Duration } \\
\text { (years) }\end{array}$ & $\begin{array}{l}\text { Pure tone } \\
\text { audiometric } \\
\text { threshold (dB HL)* }\end{array}$ & $\begin{array}{l}\text { Tinnitus loudness } \\
\text { (dB HL) }\end{array}$ & $\begin{array}{l}\text { Tinnitus frequency } \\
(\mathrm{kHz}) \text { and type }\end{array}$ & $\begin{array}{l}\text { Audiometric } \\
\text { threshold at } \\
\text { tinnitus (dB HL) }\end{array}$ & Stimulation site & $\begin{array}{l}\text { rTMS intensity } \\
(\%)\end{array}$ \\
\hline 1 & $49 / \mathrm{m}$ & 4 & $\mathrm{r}: 12, \mathrm{l}: 13, \mathrm{hf}$ & $\mathrm{r}: 35, \mathrm{l}: 49$ & $8, \mathrm{SIN}$ & r: 32, I: $41:$ & I BA 22 & 82 \\
\hline 2 & $59 / \mathrm{m}$ & 10 & r: 55 , I: 51 , pan & r: 60, l: 56 & $3, \mathrm{SIN}$ & $r: 56$, l: 53 & I BA 39 & 74 \\
\hline 3 & $58 / \mathrm{m}$ & 2 & $\mathrm{r}: 6, \mathrm{l}: 7, \mathrm{hf}$ & r: 43, I: 58 & $10, \mathrm{SIN}$ & r: 40, I 53: & r BA 39 & 58 \\
\hline 4 & $68 / \mathrm{m}$ & 4 & $\mathrm{r}: 6, \mathrm{l}: 6, \mathrm{hf}$ & r: 22, I: 27 & 6, SIN & r: 21, I: 26 & r BA 22 & 55 \\
\hline 5 & $59 / \mathrm{m}$ & 10 & r: $69,1: 22$, pan & r: $75, \mathrm{I}:$ no & $3, \mathrm{NBN}$ & r: $70, \mathrm{l}:-$ & r BA 39 & 91 \\
\hline 6 & $53 / f$ & 4 & $\mathrm{r}: 15, \mathrm{l}: 11, \mathrm{hf}$ & r: 69, I: 62 & $10, \mathrm{SIN}$ & r: $55, \mathrm{l}: 61$ & I BA 39 & 77 \\
\hline
\end{tabular}

BA, Brodmann area; $f$, female; hf, high-frequency hearing loss; $\mathrm{HL}$, hearing level; I, left; $\mathrm{m}$, male; NBN, narrow band noise; no, not obtained; pan, pancochlear hearing loss; $r$, right; SIN, sinus tone.

*HL, hearing level in decibel $(\mathrm{dB})$, provided as a standard "four tone average" = hearing loss at $((0.5+1+2+3 \mathrm{kHz}) / 4)$. 
statistically analysed by using the Friedman analysis of variance and a retrospective Wilcoxon non-parametric test (two sided) for paired samples. In addition, tinnitus was evaluated by three self-assessment scales on tinnitus loudness, annoyance and global tinnitus change similar to those described by Zenner and De Maddalena. ${ }^{27}$ The tinnitus-change score (TCS) was applied to rate the subjective effect of the intervention on tinnitus using a six-point scale (3, tinnitus disappeared; 2, greatly improved; 1 , improved; 0 , unchanged; -1 , worse; and -2 , worst). ${ }^{27}$ This was carried out after every 2 week course of treatment (verum and sham). Additionally, after each stimulation, tinnitus loudness and annoyance were rated on a six-point and an eight-point response scale, respectively. ${ }^{27}$

To find brain regions with tinnitus-related neural activity predictive for rTMS response, ?rCBF images were stereotactically normalised, smoothed and statistically analysed with SPM2 (linear regression: $\triangle \mathrm{TQ} v$ ?rCBF, voxel-level threshold: $\mathrm{p}<0.001)$. Clusters that survived SPM correction for multiple comparisons were depicted as masked contrast. ${ }^{28}$

\section{RESULTS}

\section{Clinical findings}

Table 1 summarises the demographic data, audiological characteristics and rTMS parameters for each patient.

\section{Effects of rTMS on tinnitus questionnaire score (tinnitus- related distress)}

No side effects of stimulation were observed or reported by the patients.

A 2-week treatment with low-frequency rTMS, guided to the individual area of maximum tinnitus-related activation in the temporoparietal cortex, exerted an attenuation on tinnitusrelated distress, as shown by non-parametric analysis of variance (Friedman ANOVA CONDITION $^{2} \chi^{2}=8.0 ; p=0.022$ ) on the tinnitus questionnaire score. Direct comparison indicated a reduction in the tinnitus questionnaire score after verum stimulation compared with baseline and control stimulation $(\mathrm{Z}=-2.032 ; \mathrm{p}=0.042)$. Two weeks after treatment, tinnitus distress returned to baseline in all patients but one (fig 1 ).

\section{Effects of rTMS on TCS (global change)}

Regarding the global tinnitus change as quantified by the TCS, one subject reported the tinnitus as "improved" and another as "greatly improved" after 2 weeks of verum rTMS. Four subjects did not appraise the tinnitus as changed on the TCS.

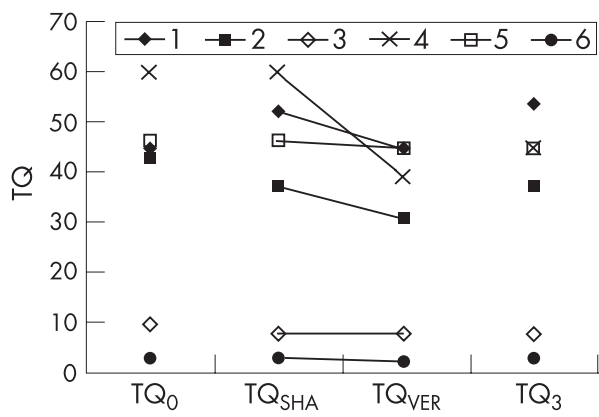

Figure 1 Tinnitus questionnaire score at baseline $\left(T_{0}\right)$, after repetitive transcranial magnetic stimulation to the temporoparietal maximum of tinnitus-related hyperactivity (TQ 2 weeks after the end of treatment $\left(\mathrm{TQ}_{3}\right)$. Table 2 gives the order of treatment conditions.

\section{Effects of rTMS on tinnitus loudness and annoyance}

Table 2 lists the additional ratings of tinnitus loudness and annoyance. Both the self-assessment scales for tinnitus loudness and tinnitus annoyance showed a tendency towards a benefit of rTMS (median tinnitus loudness scale 4, and median tinnitus annoyance 4) versus placebo stimulation (medians 4.5 and 4.5) when compared with baseline (medians 5 and 5).

\section{Correlation of tinnitus-related rCBF changes and rTMS effects}

In the SPM analysis (fig 2), we found one cluster with significant negative correlation between $\triangle \mathrm{rCBF}$ and $\triangle \mathrm{TQ}$ (ie pronounced tinnitus-related hyperactivity corresponds to a good response to rTMS), located in the anterior cingulate $\left(\mathrm{T}_{\max }=23.4, \mathrm{CON}_{\max }\right.$ at MNI $[-4 /+52 / 0], 154$ voxel $\left.=1.2 \mathrm{ml}\right)$.

\section{DISCUSSION}

In this study, repeated sessions of rTMS guided to the individual maximum of tinnitus-related cortical activity induced a moderate reduction of tinnitus. Notably, interindividual variability was high. Compared with sham stimulation, tinnitus distress as quantified by the tinnitus questionnaire score was reduced in five of six patients. However, only in the two subjects with the highest reduction of tinnitus questionnaire score $(\geqslant 40 \%)$ would we consider the improvements clinically relevant, as indicated in the self-assessment scores of tinnitus change, loudness and annoyance.

These results are an extension of our earlier studies showing the short-term effects of high-frequency and low-frequency rTMS on tinnitus, and point towards a pivotal role for the secondary and integrative auditory cortical areas. ${ }^{12} 17$ Furthermore, they extend findings from studies on the effects of repeated sessions of rTMS aimed towards the primary auditory cortex in tinnitus ${ }^{20} 2129$ in several ways. In our study: (1) rTMS was directed to cortical areas specifically active during tinnitus perception as assessed by functional imaging (ie higher-order association areas (BA 39, BA 22)); (2) control stimulation simulated loudness and the aversive sensation of real rTMS; (3) the course of treatment was extended to 2 weeks; (4) a score of tinnitus change was obtained to shed light on the clinical relevance of this effect; and (5) functional imaging data were used to identify areas with potential influence on rTMS response.

Limitations of the study, particularly the placebo control, are the crossover design and the fact that the subjects had already participated in a study on rTMS in tinnitus. However, subjects were not informed about one of the two conditions being ineffective, and the study was conducted several months after the previous experiments.

Previous literature on the neurophysiology of tinnitus focused on changes in the auditory system in the narrower sense. Nevertheless, several imaging studies showed activation of higher-order association areas and the limbic system during tinnitus perception. ${ }^{10}{ }^{12}{ }^{30}$ Interference with neuronal function in temporoparietal cortical regions can transiently suppress tinnitus, pointing to the involvement of these areas in tinnitus perception. ${ }^{17}{ }^{18}{ }^{31}$ Moreover, the attentional and emotional state is tightly connected with tinnitus perception and the related distress. ${ }^{32}{ }^{33}$ Hence, evidence is accumulating that tinnitusrelated neuronal reorganisation occurs at various stages of the hierarchical auditory processing, including cortical areas of multimodal association and emotional evaluation. Interestingly, in agreement with earlier studies, ${ }^{10}{ }^{11}$ our previous data $^{12}$ did not support findings of enhanced $\mathrm{rCBF}$ in the primary auditory cortex. This is in line with a study on animals showing that an acute noise trauma (assumed to induce tinnitus) is not immediately followed by an increase in firing rates in the 
Table 2 Effects of repetitive transcranial magnetic stimulation on tinnitus

\begin{tabular}{|c|c|c|c|c|c|c|c|c|c|c|c|c|}
\hline Patient & Order & $\mathrm{TQ}_{0}$ & $\Delta \mathrm{TQ}(\%)^{*}$ & $\mathrm{TQ}_{3}$ & TCS $_{\text {VER }}$ & $\mathrm{TCS}_{\mathrm{SHA}}$ & $\mathrm{TL}_{0}$ & $\mathrm{TL}_{\text {VER }}$ & $\mathrm{TL}_{\text {SHA }}$ & $\mathrm{TA}_{0}$ & $T_{\text {VER }}$ & $\mathrm{TA}_{\mathrm{SHA}}$ \\
\hline 1 & $v-p$ & 45 & -17.70 & 54 & 0 & 0 & 5 & 5 & 5 & 5 & 6 & 5,5 \\
\hline 2 & $v-p$ & 43 & -14.40 & 37 & 0 & 0 & 5 & 5 & 5 & 5 & 5 & 5 \\
\hline 3 & $p-v$ & 10 & 0 & 8 & 0 & 0 & 5 & 3 & 3,5 & 4 & 3 & 2,5 \\
\hline 4 & $p-v$ & 60 & -42.40 & 45 & -2 & -1 & 5 & 1 & 2 & 5 & 1 & 3 \\
\hline 5 & $p-v$ & 46 & -2.2 & 45 & 0 & 0 & 5 & 6 & 6 & 6 & 6 & 6 \\
\hline 6 & $v-p$ & 3 & -40 & 3 & -1 & 0 & 4 & 3 & 4 & 4 & 3 & 4 \\
\hline
\end{tabular}

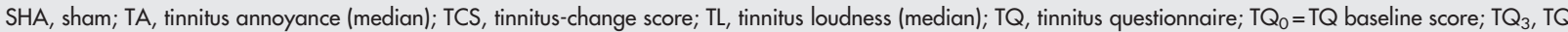
score 2 weeks after treatment; VER, verum.

${ }^{*} \Delta T Q=100 \% \times\left(T Q_{V E R}-T Q_{S H A}\right) /\left(\left(T Q_{V E R}+T Q_{S H A}\right) / 2\right)$.
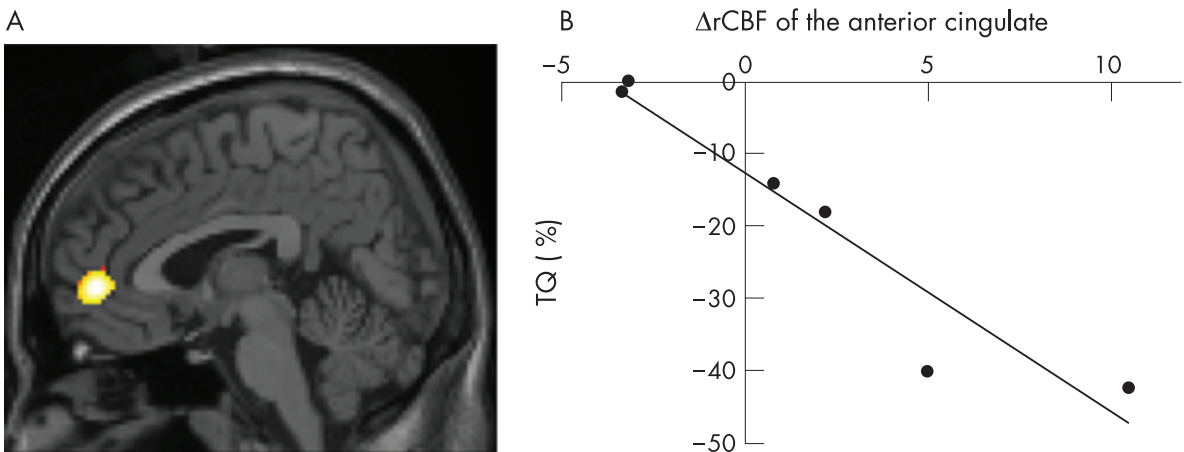

Figure 2 Explorative statistical parametric mapping (SPM) analysis of tinnitus-related change in regional cerebral blood flow ( $\triangle \mathrm{rCBF}$ ) in comparison with treatment response $\triangle T Q$ : (A) A cluster with negative correlation (tinnitus-related hyperactivity associated with good response) was found in the anterior cingulate and adjacent BA10, surviving correction of multiple comparisons $\left(P_{\text {corr }}=0.004\right)$. Colour codes reflect $\Delta r C B F$, masked with a "regional least significant difference" derived from SPM analysis. (B) Individual data, extracted from the cluster in the anterior cingulate.

primary auditory cortex, ${ }^{15}$ but does not generally preclude maladaptive changes of function in these areas.

In terms of selecting the optimal target region for rTMS, we assumed that interfering with tinnitus-related hyperactivity by rTMS would be most effective. In fact, this approach seemed to be feasible, although access to PET facilities is often limited and a considerable group of non-responders to rTMS remain. The lack of response in some subjects might have several reasons: (1) a focus of hyperactivity situated in a cortical sulcus may be out of range of rTMS; (2) between-subject variability of response to rTMS as documented in the motor cortex ${ }^{34}$; and (3) the distribution and accessibility of the tinnitus-related neuronal network varies between subjects and might be related to the duration of tinnitus history. ${ }^{12} 18$

A high tinnitus-related neuronal activity $(\triangle \mathrm{rCBF})$ in the anterior cingulate gyrus (ACC) correlated with a good response to rTMS. Although our sample size was limited, the cluster survived SPM's correction for multiple testing, justifying the hypothesis of ACC- $\triangle \mathrm{rCBF}$ being a predictor of response to rTMS. This is not implausible, as this area is involved in auditory attention ${ }^{35}$ and emotional control of aversive stimuli and pain. ${ }^{36}{ }^{37}$ These findings extend our previous observation ${ }^{12}$ of enhanced tinnitus-related activity in the posterior cingulate cortex and correspond to a model of differential functions of the cingulate cortex. ${ }^{36}$ In this model, the posterior cingulate cortex is involved in evaluative monitoring and memorisation of sensory input, whereas the ACC represents an executive region related to emotional control of response to internal or external stimuli.

Our findings do not provide clear-cut evidence for a reduction of tinnitus outlasting the course of treatment. There is no significant difference between the tinnitus-related distress at baseline and 2 weeks after the end of treatment with target rTMS. Tinnitus remained unchanged in two patients (one of them was a non-responder) but increased again in four. Nevertheless, the patient with the greatest reduction in tinnitus continued to show some decreased tinnitus-related distress. In contrast with the study of Kleinjung et al, ${ }^{21}$ our findings suggest that the effects of repeated rTMS sessions on tinnitus wear off with time. In light of corresponding neurophysiological data, this seems to be plausible. ${ }^{19}$ Long-term depression-like effects are considered to be reversible and do not increase over months after stimulation.

It should be mentioned that May et $a l^{38}$ generated initial evidence that five sessions of rTMS applied to the superior temporal cortex might induce macroscopic changes in the auditory cortex. This finding may represent a neurophysiological substrate of persisting changes of cortical function. However, at present, the neuronal underpinnings are unclear. An alternative to prolonged rTMS for the treatment of chronic tinnitus has been suggested with long-term electrical stimulation of the primary or secondary through auditory cortex implantation. ${ }^{39}$

In conclusion, repeated sessions of rTMS are effective in decreasing tinnitus-related distress in a subgroup of patients during the course of treatment. Although the magnitude of reduction is only moderate, this finding indicates that noninvasive or invasive brain stimulation may provide an interesting new option for a neurophysiology-based treatment of chronic tinnitus.

\section{Authors' affiliations}

C Plewnia, Brain-Stimulation Laboratory, Department of Psychiatry, University of Tuebingen, Tuebingen, Germany

M Reimold, Department of Nuclear Medicine, PET Center, University of Tuebingen, Tuebingen, Germany

A Najib, Hertie-Institute for Clinical Brain Research, Department of General Neurology, Cortical Physiology Research Group, University of Tuebingen, Tuebingen, Germany 
G Reischl, Department of Nuclear Medicine, Radiopharmacology Section, University of Tuebingen, Tuebingen, Germany

S K Plontke, Department of Otorhinolaryngology-Head and Neck Surgery, Tuebingen Hearing Research Center, University of Tuebingen, Tuebingen, Germany

C Gerloff, Department of Neurology, Center of Clinical Neurosciences, University Medical Center Hamburg-Eppendorf, Hamburg, Germany

Funding: This study was funded by grants from the American Tinnitus Association (ATA) and the University of Tuebingen (fortuene-program 1216-0-0). These funding sources had no involvement in the study design; in the collection, analysis and interpretation of data; in the writing of the report; and in the decision to submit the paper for publication.

Competing interests: None.

\section{REFERENCES}

1 Lockwood AH, Salvi RJ, Burkard RF. Tinnitus. N Engl J Med 2002:347:904-10.

2 Heller AJ. Classification and epidemiology of tinnitus. Otolaryngol Clin North Am 2003;36:239-48.

3 Axelsson A, Ringdahl A. Tinnitus-a study of its prevalence and characteristics. Br J Audiol 1989;23:53-62.

4 Dobie RA. Depression and tinnitus. Otolaryngol Clin North Am 2003:36:383-8.

5 Folmer RL, Carroll JR. Long-term effectiveness of ear-level devices for tinnitus. Otolaryngol Head Neck Surg 2006;134:132-7.

6 Vernon JA. Masking of tinnitus through a cochlear implant. J Am Acad Audiol 2000:11:293-4.

7 Jastreboff PJ, Jastreboff MM. Tinnitus retraining therapy (TRT) as a method for treatment of tinnitus and hyperacusis patients. J Am Acad Audiol 2000;11:162-77

8 Hiller W, Haerkotter C. Does sound stimulation have additive effects on cognitivebehavioral treatment of chronic tinnitus? Behav Res Ther 2005:43:595-612

9 Mirz F, Pedersen B, Ishizu K, et al. Positron emission tomography of cortical centers of tinnitus. Heart Res 1999;134:133-44.

10 Giraud AL, Chery-Croze S, Fischer G, et al. A selective imaging of tinnitus. Neuroreport 1999; 10:1-5.

11 Reyes SA, Salvi RJ, Burkard RF, et al. Brain imaging of the effects of lidocaine on tinnitus. Hear Res 2002;171:43-50.

12 Plewnia C, Reimold M, Najib A, et al. Dose-dependent attenuation of auditory phantom perception (tinnitus) by PET-guided repetitive transcranial magnetic stimulation. Hum Brain Mapp 2006. Published online first: 13 Jun 2006, doi: $10.1002 / \mathrm{hbm} .20270$

13 Lockwood AH, Salvi RJ, Coad ML, et al. The functional neuroanatomy of tinnitus: evidence for limbic system links and neural plasticity. Neurology 1998;50:114-20.

14 Lockwood AH, Wack DS, Burkard RF, et al. The functional anatomy of gazeevoked tinnitus and sustained lateral gaze. Neurology 2001;56:472-80.

15 Norena AJ, Eggermont JJ. Changes in spontaneous neural activity immediately after an acoustic trauma: implications for neural correlates of tinnitus. Heart Res 2003; 183:137-53.

16 Eggermont JJ, Roberts LE. The neuroscience of tinnitus. Trends Neurosci 2004; 27:676-82.

17 Plewnia C, Bartels M, Gerloff C. Transient suppression of tinnitus by transcranial magnetic stimulation. Ann Neurol 2003;53:263-6.

18 De Ridder D, Verstraeten E, Van der Kelen K, et al. Transcranial magnetic stimulation for tinnitus: influence of tinnitus duration on stimulation parameter choice and maximal tinnitus suppression. Otol Neurotol 2005;26:616-19.
19 Chen R, Classen J, Gerloff C, et al. Depression of motor cortex excitability by low-frequency transcranial magnetic stimulation. Neurology 1997;48:1398-403.

20 Eichhammer $\mathbf{P}$, Langguth B, Marienhagen J, et al. Neuronavigated repetitive transcranial magnetic stimulation in patients with tinnitus: a short case series. Biol Psychiatry 2003;54:862-5.

21 Kleinjung T, Eichhammer $P$, Langguth $B$, et al. Long-term effects of repetitive transcranial magnetic stimulation (rTMS) in patients with chronic tinnitus. Otolaryngol Head Neck Surg 2005;132:566-9.

22 Feldmann H. Specific audiological diagnostics. In: Feldmann H, eds. Tinnitus. 2nd edn. Stuttgart: Thieme, 1998.

23 Henry JA, Meikle MB. Psychoacoustic measures of tinnitus. Am Acad Audiol 2000;11:138-55

24 Kroner-Herwig B, Hebing G, van Rijn-Kalkmann U, et al. The management of chronic tinnitus-comparison of a cognitive-behavioural group training with yoga. $J$ Psychosom Res 1995; 39:153-65.

25 Goebel G, Hiller W. The tinnitus questionnaire. A standard instrument for grading the degree of tinnitus. Results of a multicenter study with the tinnitus questionnaire. HNO 1994;42:166-72.

26 Hallam RS, Jakes SC, Hinchcliffe R. Cognitive variables in tinnitus annoyance. Br J Clin Psychol 1988:27:213-22.

27 Zenner HP, De Maddalena H. Validity and reliability study of three tinnitus selfassessment scales: loudness, annoyance and change. Acta Otolaryngol 2005; 125:1184-8.

28 Reimold $M$, Slifstein $M$, Heinz A, et al. Effect of spatial smoothing on t-maps: arguments for going back from t-maps to masked contrast images. J Cereb Blood Flow Metab 2006;26:751-9.

29 Langguth B, Eichhammer P, Wiegand R, et al. Neuronavigated rTMS in a patient with chronic tinnitus. Effects of 4 weeks treatment. Neuroreport 2003; 14:977-80.

30 Gardner A, Pagani $M$, Jacobsson $H$, et al. Differences in resting state regional cerebral blood flow assessed with 99mTc-HMPAO SPECT and brain atlas matching between depressed patients with and without tinnitus. Nucl Med Commun 2002;23:429-39.

31 Fregni F, Marcondes R, Boggio P, et al. Transient tinnitus suppression induced by repetitive transcranial magnetic stimulation and transcranial direct current stimulation. Eur J Neurol 2006;13:996-1001.

32 Newman CW, Wharton JA, Jacobson GP. Self-focused and somatic attention in patients with tinnitus. J Am Acad Audiol 1997;8:143-9.

33 Folmer RL, Griest SE, Martin WH. Chronic tinnitus as phantom auditory pain Otolaryngol Head Neck Surg 2001;124:394-400.

34 Touge T, Gerschlager W, Brown P, et al. Are the after-effects of low-frequency rTMS on motor cortex excitability due to changes in the efficacy of cortical synapses? Clin Neurophysiol 2001;112:2138-45.

35 Sturm W, de Simone A, Krause BJ, et al. Functional anatomy of intrinsic alertness: evidence for a fronto-parietal-thalamic-brainstem network in the right hemisphere. Neuropsychologia 1999;37:797-805

36 Vogt BA, Finch DM, Olson CR. Functional heterogeneity in cingulate cortex: the anterior executive and posterior evaluative regions. Cereb Cortex 1992;2:435-43.

37 Shibasaki H. Central mechanisms of pain perception. Supp/ Clin Neurophysiol 2004;57:39-49.

38 May A, Hajak G, Ganssbauer S, et al. Structural brain alterations following 5 days of intervention: dynamic aspects of neuroplasticity. Cereb Cortex 2006 Published online first: 15 Feb 2006, doi: 10.1093/cercor/bhj138.

39 De Ridder D, De Mulder G, Verstraeten E, et al. Primary and secondary auditory cortex stimulation for intractable tinnitus. J Otorhinolaryngol Relat Spec $2006 ; 68: 48-55$ 